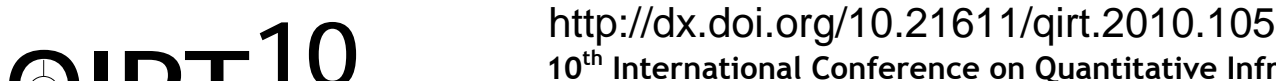 \\ $10^{\text {th }}$ International Conference on Quantitative InfraRed Thermography \\ July 27-30, 2010, Québec (Canada)
}

\section{Using ultrasound infrared thermography to detect defects in pipes}

\author{
by H. S. Park*, M. Y. Choi*, J. H. Park and S. S. Lee*
}

*Safety Measurement Center, Korea research institute of standards and science(Korea), mychoi@kriss.re.kr

\begin{abstract}
The applicability of ultrasound infrared thermography method for real-time crack detection is described. in this technique, an ultrasound wave passing through a material with mechanical discontinuity, such as a crack of disbond, causes temperature raise of that due to internal friction or thermo-mechanical effect. And the elastic wave with vibration energy is converted to the heat in the vicinity of the crack of the defect. It is local heat emitted in the crack of the defect is monitored as the hot spot area via the thermal infrared camera. As defects are usually correlated with an enhanced loss angle, ultrasound infrared lock-in thermography (ULT) is a defect selective NDE method. In this paper we investigate the applicability of ULT for maintenance and inspection. The test material was austenitic STS 304, Which is used as pipelines in the reactor coolant system of a nuclear power plants. Internal crack in a pipe using ultrasound infrared lock-in thermography imaging to detect defects were. Check direction of $0^{\circ}, 90^{\circ}, 180^{\circ}, 270^{\circ}$ in order was in progress. LPT method to obtain more specific infrared thermal imaging to determine the location of defective parts could be. At the end of the crack was able to identify hot spots.
\end{abstract}

\section{Introduction}

Currently widely used non-destructive inspection technology using infrared thermography technology, while leveraging the range is growing trend. The application of infrared thermography technology for nondestructive testing using the sample emit energy in the specimen with passive thermography impose extra energy when using the energy released in two forms of active thermography has been utilized[1,2]. In this category, ultrasound infrared thermography for the inspection object $20 \sim 30 \mathrm{kHz}$ band ultrasound to impose a defective part is inevitable in the heat, the heat source, measured by using an infrared camera to detect defects is active thermogarphy method[3]. ultrasound infrared thermography method that can scan a large area at the same time, cracks, delamination defects, such as real-time detection could be automotive, aerospace industry in the field of fault detection techniques are being utilized[4,5]. In this study, using the ultrasound infrared thermography method used in piping of nuclear power plants inside the austenite STS304 crack pipe defect detection experiment was about.

\section{2. ultrasound infrared lock-in thermography method}

While thermography generates images where the contrast is provided by local thermal emission, lock-in thermography means that one investigates a coded heat flow by analysing the temperature modulation that is induced by periodical heat deposition: As the thermal wave is reflected at the boundaries of subsurface features, its superposition to the original thermal wave causes a signal change that depends on the depth of the hidden boundary. One can show that the relevant parameter is thermal diffusion length $m$ given by

$$
\mu=\sqrt{2 \alpha / \omega}
$$

where a denotes thermal diffusivity while $\omega$ is the angular frequency of modulation: Signal magnitude is affected by boundaries in a depth that is less than $\mathrm{m}$ while signal phase still responds at about twice this depth[6-8]. 


\section{EXPERIMENTAL ARRANGEMENT}

For these experiments we used a CEDIP infrared focal plane array camera. The $320 \times 240$ detector array captures radiation in the $3 \sim 5 \mu \mathrm{m}$ spectral band at a frame rate up to $60 \mathrm{~Hz}$. A lock in module (CEDIP Altair LI) and a signal generator provided the modulated thermal source which is synchronised to the recording process of thermal images. Fig. 1 displays the corresponding experimental ULT arrangement. The ultrasonic transducer is attached to the component which is monitored by a lock-in thermography system tuned to the low frequency of amplitude modulation. The elastic wave frequency was typically around $19.8 \mathrm{KHZ}$ while the amplitude modulation frequency was usually below $50 \mathrm{mHz}$. The acoustic energy provided by the source was in most experiments 420 watts.

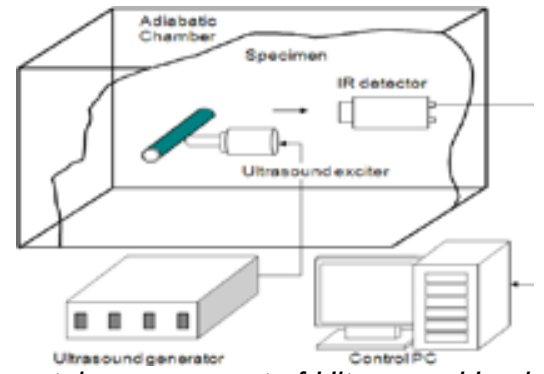

Fig. 1 Experimental arrangement of Ultrasound Lock-in Thermography

\section{Result}

$90^{\circ}$ increments rotating pipe, and when taking a flaw was detected signal. Check direction of $0^{\circ}, 90^{\circ}, 180^{\circ}, 270^{\circ}$ in order was in progress. LPT method to obtain more specific infrared thermal imaging to determine the location of defective parts could be. At the end of the crack was able to identify hot spots. In defects located in the direction of the circumference were able to identify.

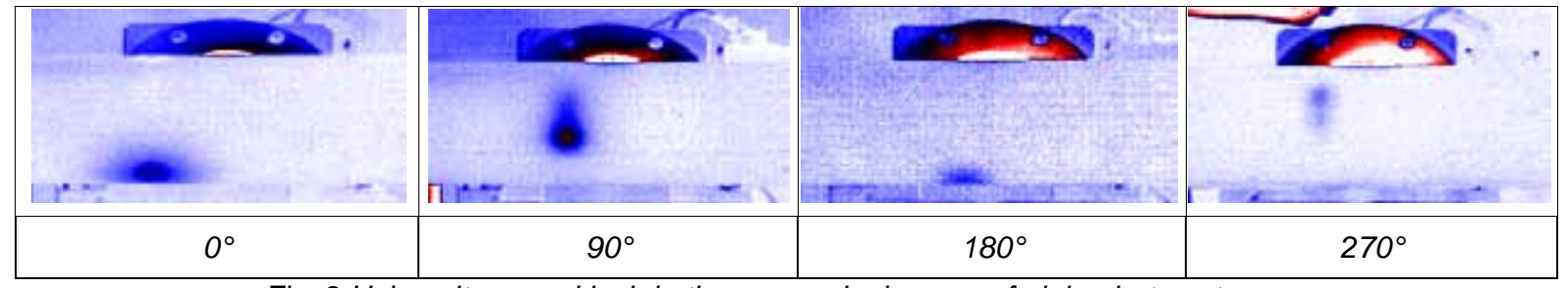

Fig. 2 Using ultrasound lock-in thermography images of piping hot spot

\section{REFERENCES}

[1] Mignogna, R. B., Green, R. E.J r., Duke, J . C. J r., Henneke, EGII., Reifsnider, K. L., "Thermographic investigation of high-power ultrasonic heating in materials", Ultrasonics, (USA), Vol. 19, p. 159-163, 1981

[2] Zong, Mingcheng., Zhang, J ianxin. and Zhao, Yan., "Pulse-heating infrared thermography non-destructive testing technique", SPIE, (China), Vol. 2899, p. 654-659, 1996

[3] Dillenz, A., Salerno, A., Wu, D., Rantala, J . and Busse, G., "P rogress in ultrasonic lockin thermography", QIRT 98,(Germany), p. 154-160, 1998

[4] Busse, G., Wu, D., and Karpen, W., "Thermal wave imaging with phase sensitive modulated thermpgraphy", J . Appl. Phys., (Germany), Vol. 71, No. 8, p. 3962-3965, 1992

[5] M. Rafet, et al, "Assessment of testicular core temperatures using microwave thermography", Human Reproducion, (UK), Vol. 15, No. 8, p. 1723-1726, 2000

[6] Busse, G., "Optoacoustic phase angle measurement for probing a metal."Appl. Phys. (Germany),Lett.35,p.759,1979

[7] Thomas, R. L., Pouch, J. J ., Wong, Y. H., Favro, L. D. and Kuo, P. K., "Subsurface flaw detection in metals by photoacoustic microscopy." In: J . Appl. Phys. (USA), Vol. 51, p. 1152-1156, 1980

[8] Lehto, A., J a arinen, J., Tiusanen, T., J okinen, M. and Luukkala, M., " Magnitude and phase in thermal wave imaging." Electr. Lett. 17, (Finland), p.364-365, 1981 